\title{
Increased serum/plasma fibroblast growth factor 21 in type 2 diabetes mellitus: a systematic review and meta-analysis
}

\author{
Yun-Sheng Wang, ${ }^{1,2}$ Jun $\mathrm{Ye}^{2}{ }^{2}$ Yong-Hong Cao, ${ }^{2}$ Rong Zhang, ${ }^{2}$ Yan Liu, ${ }^{2}$ \\ Su-Wan Zhang, ${ }^{2}$ Wu Dai, $^{2}$ Qiu Zhang ${ }^{1}$
}

\begin{abstract}
- Additional material is published online only. To view please visit the journal online (http://dx.doi.org/10.1136/ postgradmedj-2018-136002).

1 Department of Endocrinology, The First Affiliated Hospital of Anhui Medical University, Hefei, China

${ }^{2}$ Department of Endocrinology, The Second People's Hospital of Hefei, Hefei, China
\end{abstract}

\section{Correspondence to}

Prof. Wu Dai, Department of Endocrinology, The Second People's Hospital of Hefei, Hefei 230011, China;

daiwu1984@sina.com and

Prof. Qiu Zhang, Department of Endocrinology, The First Affiliated Hospital of Anhui Medical University, Hefei, 230022, China;

zhangqiu1965@sina.com

Y-SW and JY are joint first authors.

Received 13 July 2018 Revised 15 October 2018 Accepted 27 January 2019 Published Online First 27 March 2019

\section{Check for updates}

(c) Author(s) (or their employer(s)) 2019. No commercial re-use. See rights and permissions. Published by BMJ.

To cite: Wang Y-S, Ye J, Cao Y-H, et al. Postgrad Med J 2019:95:134-139.

\section{ABSTRACT \\ Objectives Fibroblast growth factor-21 (FGF-21) plays} an important role in glucose and lipid metabolism. This study aims to systemically review the evidence regarding the relationship between the FGF-21 levels and type 2 diabetes mellitus (T2DM), as well as the related influential factors.

Methods Research related to plasma/serum FGF-21 levels in patients with T2DM and healthy controls were searched in PubMed, EMBASE and The Cochrane Library databases (up to 31 March 2017). Pooled standard mean difference (SMD) with $95 \% \mathrm{Cl}$ was calculated by fixedeffect or random-effect model analysis. Heterogeneity test was performed by the Q-statistic and quantified using $\ell^{2}$, and publication bias was evaluated using a funnel plot and Egger's linear regression test.

Results In total, 317 articles were obtained after searching databases, and 11 studies with 866 patients with T2DM and 629 controls were finally included. Metaanalysis revealed that, compared with the control group, the T2DM group had a significantly higher plasma/ serum FGF-21 level $(p<0.001)$, with the SMD of $1.34 \%$ and $95 \% \mathrm{Cl}(0.70$ to 1.98). Meta-regression analysis and subgroup analyses suggested that body mass index (BMI), triglycerides (TG) and total cholesterol (TC) were likely related to the observed FGF-21 differences between two groups.

Conclusions Overall, our study suggests that patients with T2DM have significantly higher plasma/serum FGF21 levels, and the FGF-21 levels were influenced by BMI, $\mathrm{TC}$ and $\mathrm{TG}$.

\section{INTRODUCTION}

Type 2 diabetes mellitus (T2DM) is a group of metabolic diseases characterised by hyperglycaemia resulting from resistance to insulin action and an inadequate compensatory insulin secretory response. ${ }^{12}$ The chronic hyperglycaemia of diabetes could lead to a long-term damage of pancreatic function and cause an occurrence of diabetes complication which involved in various organs/ tissue. ${ }^{2}$ To some extent, the pathogenesis of T2DM is an interaction of multiple factors, of which insulin resistance, visceral adiposity, atherogenic dyslipidaemia, endothelial dysfunction, genetic susceptibility and elevated blood pressure are contributed to the onset of T2DM. ${ }^{3}$ The proinflammation in association with visceral obesity and insulin resistance inevitably leads to the substantial production of adipocytokines. ${ }^{4}$
Fibroblast growth factor-21 (FGF-21), a recently described member of the FGF family, plays important roles in regulating endocrine function and modulating metabolic processes. ${ }^{5}$ Serum FGF-21 is secreted predominantly by the liver and, to a lesser degree, by the adipose tissue in humans. It can function as a crucial regulator mediating beneficial metabolic effects of therapeutic agents such as metformin, glucagon/glucagon-like peptide 1 analogue. ${ }^{67}$

Recent studies have disclosed the effect of FGF-21 on pancreatic islets, it could suppress cytokine-induced apoptosis in isolated rat pancreatic islets and increase insulin synthesis and promote glucose-induced insulin secretion from isolated diabetic rodent's islets. ${ }^{8} 9$ In addition, it has also been shown that treatment with FGF-21 is able to improve islet engraftment rates in experimental islet transplantation process, ${ }^{10}$ as well as improving lipoprotein profile and reducing body weight of diabetic rhesus monkeys. ${ }^{11}$ In patients with T2DM, although FGF-21 concentrations have been assessed in some studies, the results are still not appearance consistent. This disparity in different reports may be attributed to no enough sample sizes, low statistical testing power, ethnic variations and/or clinical heterogeneity. In the present study, we conducted a systematic review and meta-analysis of published literatures in order to fully estimate the FGF-21 concentrations in patients with T2DM. Furthermore, we also explored and analysed whether certain factors, such as clinical and laboratory characteristics, have an effect on FGF-21 levels in T2DM.

\section{METHODS}

\section{Search strategy}

This systematic literature review and meta-analysis is strictly subject to the Preferred Reporting Items for Systematic Reviews and Meta-Analyses (PRISMA) guidelines. ${ }^{12}$

We conducted a comprehensive literature search in electronic databases of PubMed, EMBASE and The Cochrane Library to obtain eligible studies published between database inception and 31 September 2017. We used Medical Subject Headings and other related keywords for search. The following terms were used in all fields: 'type 2 diabetes mellitus' OR 'T2DM' OR 'diabetes mellitus' AND 'fibroblast growth factor-21' OR 'fibroblast growth factor' OR 'FGF-21' OR 'FGF'. 


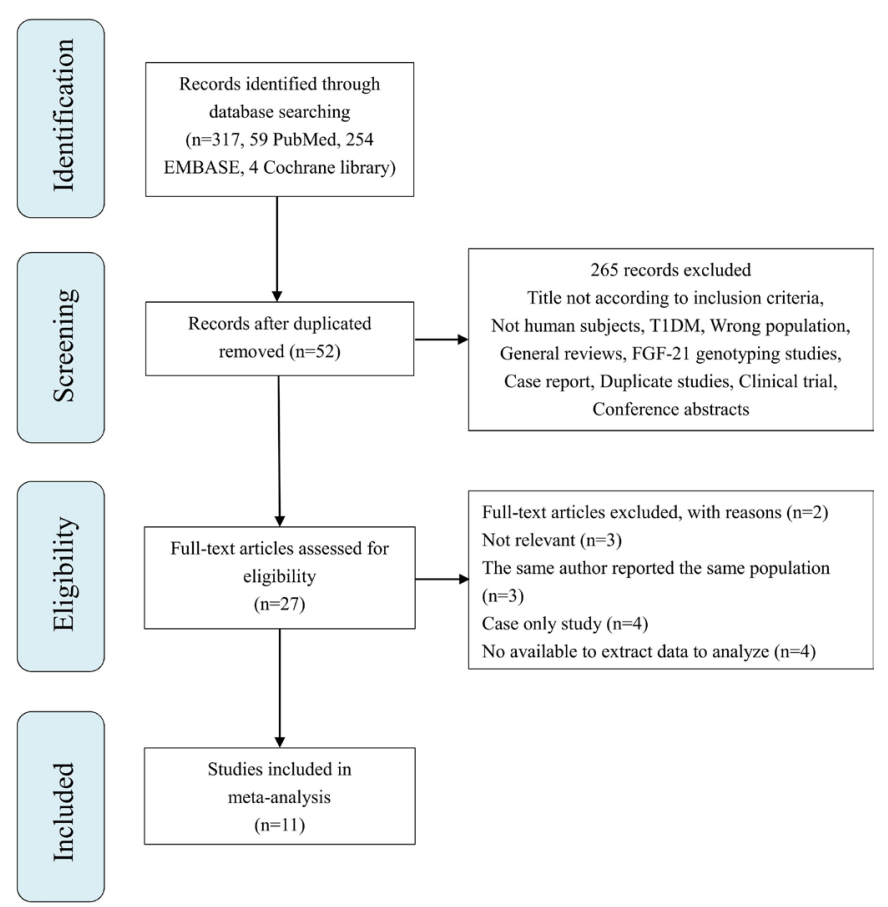

Figure 1 Flow chart of the systematic literature review. After excluding duplicate publication, unclear indicators, unmatched purposes, review and conference abstracts, 11 articles were included in the final analysis. FGF-21, Fibroblast growth factor-21; T1DM, type 1 diabetes mellitus.

All keyword variants were used to increase the number of studies returned by the search. Moreover, a manual search of reference lists was undertaken to look for additional relevant articles. There were no any language restrictions in the search strategy. If relevant information of published study was not available, we contacted the correspondence authors to obtain the additional information via email.

\section{Inclusion criteria and exclusion criteria}

For inclusion in the meta-analysis, all the following criteria had to be met: (1) published observational studies with data on both patients with T2DM and healthy age-and sex-matched controls and (2) study provided the detailed data about FGF-21 level in both cases and controls. The diagnostic criteria of T2DM were clearly defined, and healthy controls were clearly described. If study with data published more than once or using the same subjects, we considered all publications for data abstraction, only the article with adequate study strategy was chosen.

Study subjects with type 1 diabetes mellitus, pre-diabetes mellitus were excluded. In addition, studies were excluded if they were review articles, clinical trials, case report and discussion papers. We also excluded studies in which they reported ambiguous eligibility criteria. A flow chart regarding the process of studies identified, included and excluded is displayed in figure 1.

\section{Data extraction}

Quality assessment of each included studies was accessed and scored by two independent reviewers (Yun-Sheng Wang and Jun Ye) using the Newcastle-Ottawa Quality (NOS) Assessment Scale to determine the quality of selection of study participants, comparability, exposure and outcome. ${ }^{13}$ Disagreements were discussed, and further adjudication was resolved by a third reviewer (Rong Zhang).
We extracted the following data from each of included studies using a predesigned extraction form, which includes the following: first author's name, publication year, participant's age, sex proportion, body mass index (BMI), country study, study sample size (n), the concentration of FGF-21 in T2DM and controls, as well as other related information, respectively.

\section{Statistical analysis}

The effect size of mean FGF-21 difference between patients with T2DM and control was computed using standardised mean difference (SMD), and a forest plot was displayed to estimate the SMD and its $95 \%$ CIs. ${ }^{14}$ Heterogeneity of the included studies was tested by the $Q$-statistic ${ }^{15}$ and quantified using $I^{2}=100 \% \times$ $((Q$ - degree of freedom $) / Q) .{ }^{16}$ If $I^{2}$ value was more than $50 \%$ and the $p$ value for the test of heterogeneity was $<0.10$, the results were considered heterogeneous. If all the included studies shared a common effect size, then the fixed-effect model was applied. Otherwise, a random-effects model was used. Evidence of publication bias was assessed through a visual funnel plot and Egger's regression asymmetry test ${ }^{17}$ Subgroup analysis was performed to detect the underlying sources of heterogeneity. All statistical analyses were conducted by Stata V. 12.0 software.

\section{RESULTS}

\section{Study characteristics}

Our initial search, from PubMed, EMBASE and The Cochrane Library databases, yielded 317 studies. In all, 290 articles were excluded from the study due to the following reasons: (1) not original articles, (2) not English articles, (3) duplicated articles, (4) case only studies, (5) not pertaining to T2DM, (6) not pertaining to FGF-21 and (7) no available data. Of the other 27 potential articles, 11 articles including 1495 subjects $(866$ patients with T2DM and 629 healthy controls) fulfilled all the inclusion criteria and were ultimately incorporated in this meta-analysis. The basic characteristics of included studies are further summarised in detail in Table $1 .{ }^{18-28}$ FGF-21 concentration was compared between patients with T2DM and age-, sex- and BMI-matched healthy controls in each included study. Quality assessment by NOS was determined among each included studies (online supplementary table 1) (table 1). The scores of all the included studies ranged from 5 to 8 stars and were considered a relative high quality.

\section{Meta-analysis results}

\section{Heterogeneity test results}

Heterogeneity was tested with Cochran's $Q$-statistic and quantified using $I^{2}$. In this study, the heterogeneity across studies was statistically significant $\left(I^{2}=96.50 \%, \mathrm{p}<0.001\right)$ (figure 2; meta-analysis of 11 studies reporting on FGF-21 in T2DM compared with controls).

\section{Overall effects}

Overall, the incorporate analysis showed that patients with T2DM have significantly higher levels of FGF-21 for a comparison of healthy controls (SMD $=1.34,95 \%$ CI: 0.70 to 1.98 ) (figure 2).

\section{Meta-regression analysis and subgroup analysis}

Meta-regression analysis was conducted to explore the potential heterogeneity sources. The effects of covariates on FGF-21 are shown in table 2, and the results showed that BMI, triglycerides (TG) and total cholesterol (TC) were all statistically significant covariates and associated with observed FGF-21 differences 


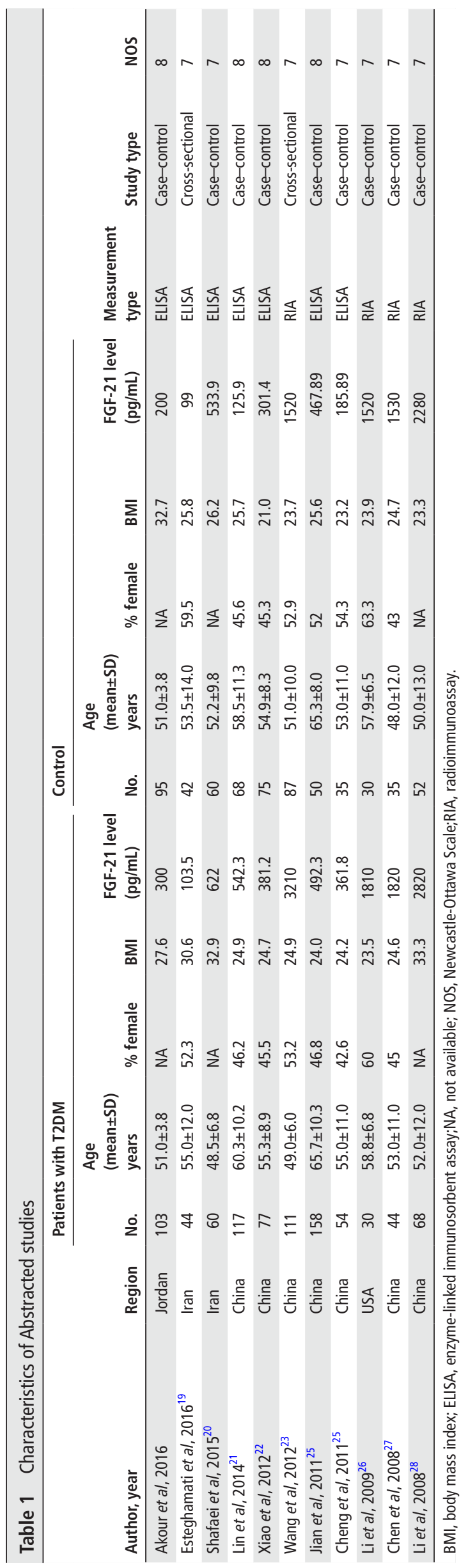

within study groups (all $\mathrm{p}<0.05$ ). However, no such association was observed for publication year, NOS score, measurement type, total sample size, high-density lipoprotein (HDL) and low-density lipoprotein (LDL). It suggested that BMI, TG and TC are the main effects of covariates contributing to the between-study heterogeneity.

Given the existence of heterogeneity, we also performed subgroup analysis, based on BMI, race, measurement options, TG, TC, HDL and LDL, to identify the possible source of heterogeneity across the studies. The lowest FGF-21 levels among those factors was in TG $<150 \mathrm{mg} / \mathrm{dL}(0.29$ (95\% CI: 0.06 to 0.52$)$ ) and the highest was $110 \mathrm{mg} / \mathrm{dL} \leq \mathrm{TC}<220 \mathrm{mg} / \mathrm{dL}$ (2.90, 95\% CI: 0.92 to 4.02$)$ followed by BMI $\geq 25$ (2.22, 95\% CI: 0.42 to 4.02 ) (table 3 ). It suggested that BMI and TC were positively associated with FGF-21 in T2DM, whereas TG was negatively associated with FGF-21 in T2DM.

\section{Publication bias}

Publication bias was visually depicted by a funnel plot, which showed an unsymmetrical shape. Later, Egger's linear regression test was applied to statistically assess the asymmetry of the funnel plot, ${ }^{29}$ and the results suggested that there was no significant publication bias as a whole $(\mathrm{t}=1.53, \mathrm{p}=0.161)$ (figure 3$)$.

\section{Sensitivity analyses}

Sensitivity analysis was performed through the leave-one-out analyses that assessed the key studies that had substantial impact on between-study heterogeneity. The results showed no significant change when each study was withdrawn separately ( $p>0.05$ ), suggesting that the results of this meta-analysis were stabilised in FGF-21 levels between patients with T2DM and controls (figure 4).

\section{DISCUSSION}

FGF-21, as a recently identified polypeptide hormone, is a novel member of FGF family. It is preferentially expressed in liver and has been identified as a potent regulator of glucose and lipid homeostasis. ${ }^{30}$ Numerous animal-based studies have documented that FGF-21 possesses potent beneficial effects on glucose and lipid metabolism and insulin sensitivity without obvious adverse effects. ${ }^{1131}$ Recent human studies showed that serum FGF-21 levels were increased in patients with T2DM than in healthy controls; however, reported findings on the association between circulating FGF-21 and T2DM remain scant and controversial.

In this meta-analysis, we retrospectively collected the data of FGF-21 concentration from 11 comparisons of populations with T2DM and controls, a total of 1495 subjects having FGF-21 measurements reported. The results of this meta-analysis showed that there is a statistically significantly higher FGF-21 level in patients with T2DM compared with controls. Heterogeneity analysis indicated that there was a marked heterogeneity among the studies. Heterogeneity represents the degree of results variation among different studies. Strong heterogeneity may derive from the inconsistent results between-study and lead to an impaired validity of the meta-analysis. Publication bias was not detected as a whole. In order to discover the possible influences for the source of heterogeneity, the meta-regression analysis was applied. By evaluating the race, publication year, NOS score, BMI, measurement type, total sample size, TC, TG, HDL and LDL as covariates, we found that BMI, TG and TC were likely related to the observed FGF-21 difference between two groups, suggesting the potential role resulting in strong heterogeneity. 


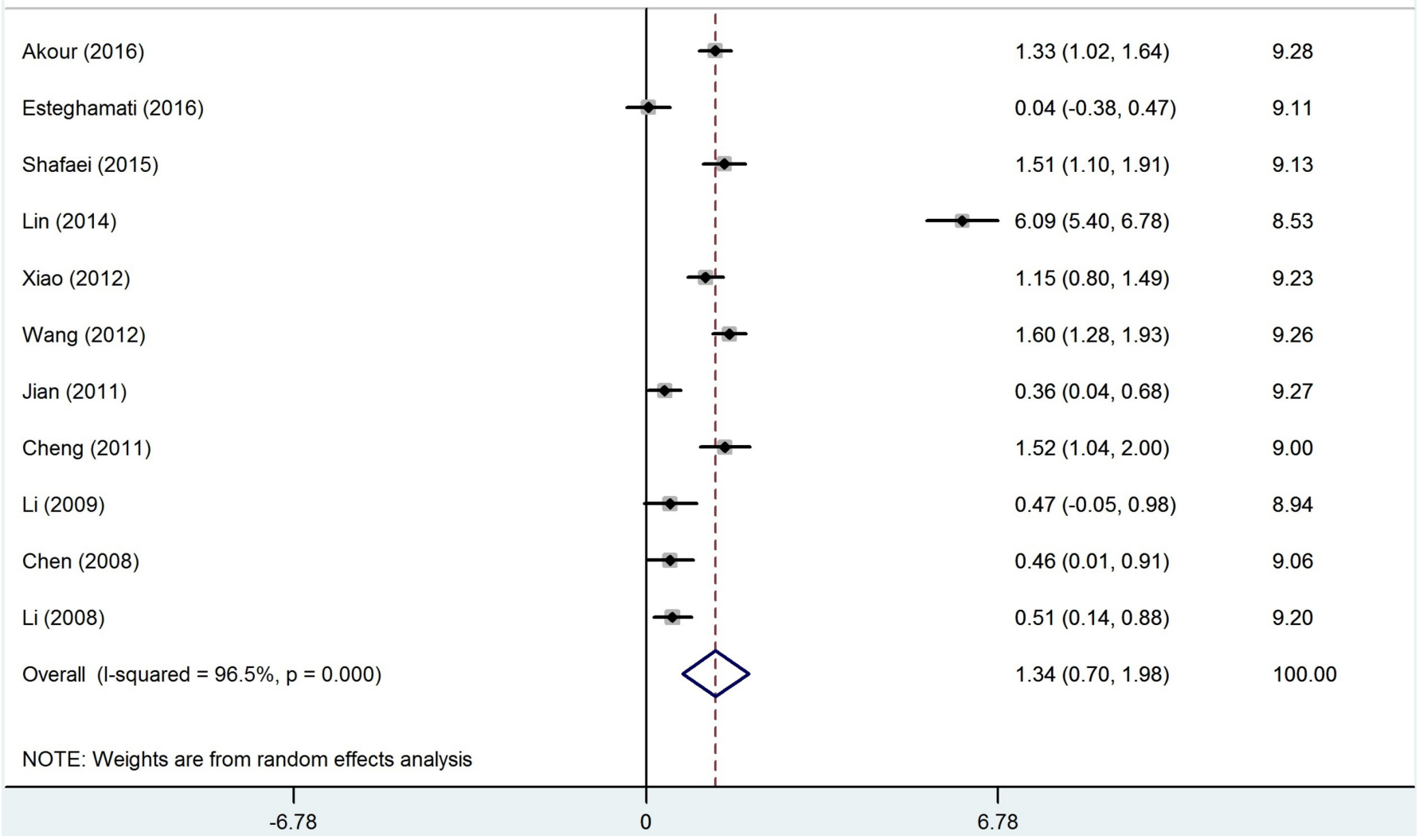

Figure 2 Forest plot of combined FGF-21 levels between patients with T2DM and controls. Random-effects meta-analysis of OPG levels between patients with T2DM and controls. FGF-21, fibroblast growth factor-21; OPG, osteoprotegerin; T2DM, type 2 diabetes mellitus.

Furthermore, the results of subgroup analyses indicated that BMI and TC were positively associated with FGF-21 in T2DM, whereas TG was negatively associated with FGF-21 in T2DM. Nevertheless, given the limited data of included studies, we cannot explore the other possible positive findings.

In the current study, there are several potential limitations that should be considered when interpreting these results. First, given the limitation of incorporated studies, of which the part of studies did not have a waist circumference (WC) report,

\begin{tabular}{|c|c|c|c|c|c|}
\hline Covariates & Coefficient & SE & $\mathrm{t}$ & $p$ Value & $95 \% \mathrm{Cl}$ \\
\hline Race & -2.734 & 1.212 & -2.26 & 0.087 & -6.100 to 0.631 \\
\hline Year & 0.219 & 0.321 & 0.68 & 0.531 & -0.067 to 1.110 \\
\hline NOS score & 1.391 & 1.263 & 1.10 & 0.332 & -2.115 to 4.898 \\
\hline BMI & 0.488 & 0.169 & 2.88 & 0.045 & 0.017 to 0.958 \\
\hline Measurement type & 1.564 & 1.450 & 1.08 & 0.341 & -2.461 to 5.590 \\
\hline Total sample size & -0.015 & 0.011 & -1.46 & 0.217 & -0.045 to 0.013 \\
\hline TG & 0.010 & 0.002 & 4.82 & 0.005 & 0.005 to 0.016 \\
\hline TC & -0.023 & 0.006 & -4.20 & 0.008 & -0.038 , to 0.091 \\
\hline HDL & -0.030 & 0.033 & -0.90 & 0.409 & -0.116 to 0.055 \\
\hline LDL & 0.007 & 0.014 & 0.52 & 0.623 & -0.029 to 0.044 \\
\hline
\end{tabular}

BMI, body mass index; HDL, high-density lipoprotein; LDL, low-density lipoprotein; NOS, Newcastle-Ottawa Quality; TC, total cholesterol; TG, triglycerides. therefore, the association between FGF-21 and WC cannot be fully understood. Second, due to the relatively low number of studies and the limited data, it may affect the stability of making conclusion. More articles with larger samples should be included to avoid potential bias. Third, the target literature search would be more intact if the efficiency of resource sharing could be improved as well to promote the credibility of the result. Fourth, different reagents and instruments for the detection of FGF-21 levels in studies included in this meta-analysis may have contributed to the heterogeneity. Furthermore, as a retrospective study, meta-analysis did not prove causation which is susceptible to the methodological deficiencies of the pooled studies.

Nevertheless, this meta-analysis also has its strengths. In compared with single-country studies, we enhanced generalisability by combining findings from 11 studies across two continents, it was able to give a more precise estimation on the relationship between FGF-21 levels and T2DM by increasing the statistical power and resolution through incorporating the results of independent analyses.

\section{CONCLUSIONS}

In the present study, we concluded that FGF-21 levels are higher in patients with T2DM than in controls, and it is influenced by BMI, TC and TG variables. However, based on the limited literatures, it is essential for us to carry out large-scale prospective 


\section{Original article}

Table 3 Subgroup analysis of FGF-21 level in T2DM

\begin{tabular}{|c|c|c|c|c|c|c|c|}
\hline \multirow[b]{2}{*}{ Stratification group } & \multirow[b]{2}{*}{$N$} & \multirow[b]{2}{*}{ SMD $(95 \% \mathrm{Cl})$} & \multicolumn{3}{|c|}{ Heterogeneity test } & \multicolumn{2}{|c|}{ Publication bias } \\
\hline & & & $Q$ & $\mathrm{p}$ Value & $I^{2}(\%)$ & $t$ & $\mathrm{p}$ Value \\
\hline Total & 11 & 1.34 (0.70 to 1.98$)$ & 286.50 & $<0.001$ & 96.50 & 1.53 & 0.161 \\
\hline \multicolumn{8}{|l|}{ BMI } \\
\hline $\mathrm{BMI} \geq 25$ & 4 & 2.22 (0.42 to 4.02$)$ & 215.84 & $<0.001$ & 98.60 & 20.29 & 0.285 \\
\hline $\mathrm{BMI}<25$ & 7 & 0.87 (0.46 to 1.29$)$ & 48.40 & $<0.001$ & 87.60 & -1.92 & 0.785 \\
\hline Combined & 11 & 1.34 (0.70 to 1.98$)$ & 286.50 & $<0.001$ & 96.50 & 1.53 & 0.161 \\
\hline \multicolumn{8}{|l|}{ Race } \\
\hline Caucasian & 4 & 0.85 (0.17 to 1.53 ) & 34.18 & $<0.001$ & 91.20 & -9.66 & 0.394 \\
\hline Non-Caucasian & 7 & 1.64 (0.68 to 2.60$)$ & 250.05 & $<0.001$ & 97.60 & 19.09 & 0.071 \\
\hline Combined & 11 & 1.34 (0.70 to 1.98$)$ & 286.50 & $<0.001$ & 96.50 & 1.53 & 0.161 \\
\hline \multicolumn{8}{|c|}{ Measurement options } \\
\hline ELISA & 7 & 1.68 (0.72 to 2.65$)$ & 252.03 & $<0.001$ & 97.60 & 18.99 & 0.068 \\
\hline RIA & 4 & 1.77 (0.55 to 1.40$)$ & 29.04 & $<0.001$ & 89.70 & -11.50 & 0.251 \\
\hline Combined & 11 & 1.34 (0.70 to 1.98$)$ & 286.50 & $<0.001$ & 96.50 & 1.53 & 0.161 \\
\hline \multicolumn{8}{|l|}{ TG } \\
\hline $\mathrm{TG}<150 \mathrm{mg} / \mathrm{dL}$ & 3 & 0.29 (0.06 to 0.52 ) & 1.94 & 0.379 & 0 & -0.16 & 0.976 \\
\hline $\mathrm{TG} \geq 150 \mathrm{mg} / \mathrm{dL}$ & 6 & 1.89 (0.83 to 2.94 ) & 217.91 & $<0.001$ & 97.70 & 17.17 & 0.158 \\
\hline Combined & 9 & 1.35 (0.57 to 2.13 ) & 282.97 & $<0.001$ & 97.20 & 1.35 & 0.220 \\
\hline \multicolumn{8}{|l|}{ TC } \\
\hline $\mathrm{TC}<110 \mathrm{mg} / \mathrm{dL}$ & 5 & 0.69 (0.17 to 1.21$)$ & 37.10 & $<0.001$ & 89.20 & -5.80 & 0.533 \\
\hline $\begin{array}{l}110 \mathrm{mg} / \\
\mathrm{dL} \leq \mathrm{TC}<220 \mathrm{mg} / \mathrm{dL}\end{array}$ & 4 & 2.90 (0.92 to 4.02 ) & 215.84 & $<0.001$ & 98.60 & 20.29 & 0.285 \\
\hline Combined & 9 & 1.35 (0.57 to 2.13 ) & 282.97 & $<0.001$ & 97.20 & 1.35 & 0.220 \\
\hline \multicolumn{8}{|l|}{$\mathrm{HDL}$} \\
\hline $\begin{array}{l}30 \mathrm{mg} / \mathrm{dL}<\mathrm{HDL}<77 \\
\mathrm{mg} / \mathrm{dL}\end{array}$ & 9 & 0.84 (0.46 to 1.21 ) & 73.95 & $<0.001$ & 89.20 & -8.00 & 0.237 \\
\hline $\mathrm{HDL} \geq 77 \mathrm{mg} / \mathrm{dL}$ & 1 & 2.09 (1.40 to 2.78 ) & 0 & - & - & - & - \\
\hline Combined & 10 & 1.32 (0.64 to 2.01 ) & 283.22 & $<0.001$ & 96.80 & 1.40 & 0.198 \\
\hline \multicolumn{8}{|l|}{ LDL } \\
\hline $\mathrm{LDL}<120 \mathrm{mg} / \mathrm{dL}$ & 7 & 0.71 (0.24 to 1.18$)$ & 61.14 & $<0.001$ & 90.20 & -5.74 & 0.504 \\
\hline $\mathrm{LDL} \geq 120 \mathrm{mg} / \mathrm{dL}$ & 3 & 1.83 (0.67 to 3.99 ) & 170.11 & $<0.001$ & 98.80 & 25.00 & 0.134 \\
\hline Combined & 10 & 1.32 (0.64 to 2.01 ) & 283.22 & $<0.001$ & 96.80 & 1.40 & 0.198 \\
\hline
\end{tabular}

BMI, body mass index;ELISA, enzyme-linked immunosorbent assay; HDL, high-density lipoprotein; LDL, low-density lipoprotein; RIA, radioimmunoassay; SMD, standard mean difference; TC, total cholesterol; TG, triglycerides.

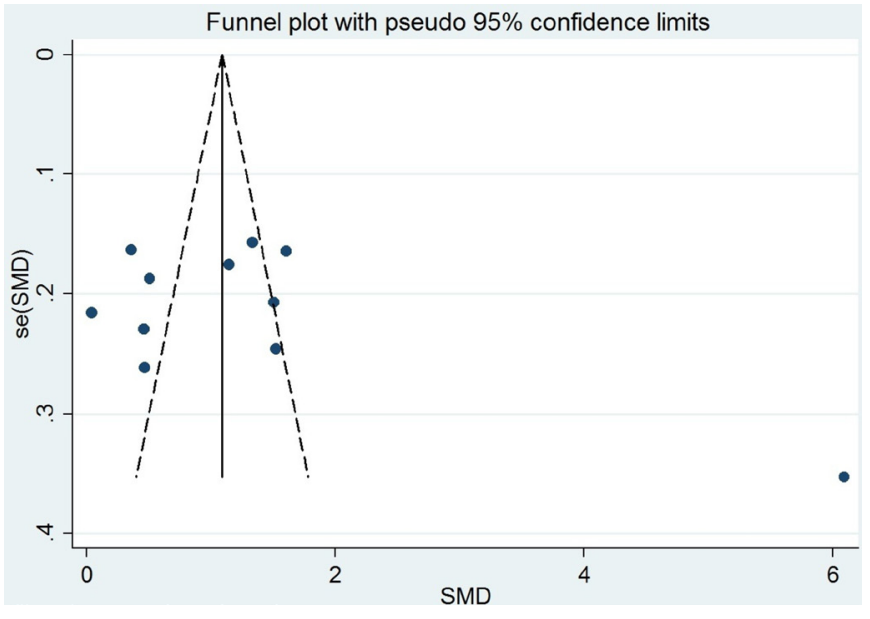

Figure 3 Funnel plot (with pseudo 95\% Cls) with the SE of the FGF21 difference plotted against the mean difference of FGF-21 of each study. Funnel plots of the relationship between the FGF-21 levels of individual studies and the precision of the study estimate. FGF-21, fibroblast growth factor-21; SMD, standard mean difference.

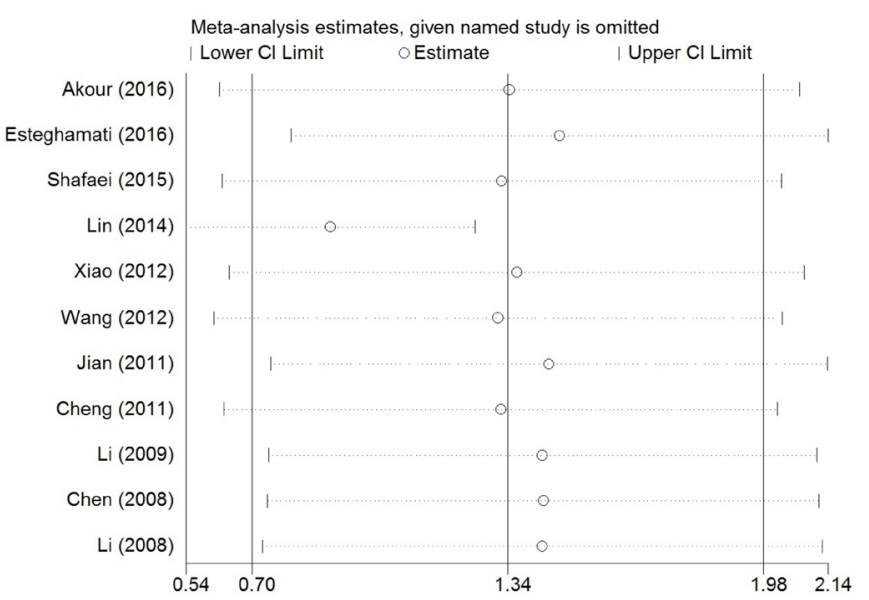

Figure 4 Sensitivity analyses by excluding one study at a time. Plot of sensitivity analysis by excluding one study each time and the pooling estimate for the rest of the studies. 
studies to determine the precise relationships between FGF-21 and T2DM.

\section{What is already known on the subject}

- Fibroblast growth factor-21 (FGF-21) plays important roles in regulating endocrine function and modulates metabolic processes.

- Recent studies have disclosed the effect of FGF-21 on pancreatic islets, it could suppress cytokine-induced apoptosis in isolated rat pancreatic islets, and increase insulin synthesis and promote glucose-induced insulin secretion from isolated diabetic rodent's islets.

- Although the expression of FGF-21 in patients with T2DM has been studied in some studies, the results are still not appearance consistent.

\section{Main messages}

- Patients with type 2 diabetes mellitus (T2DM) have a significant higher fibroblast growth factor-21 (FGF-21) level than in healthy controls.

- Increased FGF-21 levels are associated with body mass index, total cholesterol and triglycerides in patients with T2DM.

- Publication year, Newcastle-Ottawa Quality score, measurement type, total sample size, high-density lipoprotein and low-density lipoprotein do not show any relation with FGF-21 levels

\section{Current research questions}

- Further large-scale prospective studies are needed to understand the association between fibroblast growth factor-21 (FGF-21) levels and type 2 diabetes mellitus (T2DM).

- The possible regulatory effect between FGF-21 and pancreatic function.

- Based on this study, is FGF-21 can be regard as a new marker/target for the diagnosis and clinical treatment of T2DM?

Contributors YSW, WD and QZ designed the study. YSW, JY and YHC collected data, RZ and YL performed the statistical analyses. YSW and SWZ drafted the manuscript. All authors read and approved the final manuscript. YSW and JY contributed equally to this work and should be considered co-first authors.

Funding This study was supported by Anhui Science and Technology Project (grant number 1604a0802099).

Competing interests None declared.

Patient consent for publication Not required.

Provenance and peer review Not commissioned; externally peer reviewed.

\section{REFERENCES}

1 Kumana CR, Tan KCB, Cheung BMY. Absolute benefits of empagliflozin in type 2 diabetes: a game changer? Postgrad Med J 2017;93:373-5.
2 Zaccardi F, Webb DR, Yates T, et al. Pathophysiology of type 1 and type 2 diabetes mellitus: a 90-year perspective. Postgrad Med J 2016;92:63-9.

3 Donath MY, Shoelson SE. Type 2 diabetes as an inflammatory disease. Nat Rev Immunol 2011:11:98-107.

4 Shoelson SE, Lee J, Goldfine AB. Inflammation and insulin resistance. J Clin Invest 2006;116:1793-801.

5 Ornitz DM, Itoh N. Fibroblast growth factors. Genome Biol 2001;2:REVIEWS3005.

6 Dostálová I, Haluzíková D, Haluzík M. Fibroblast growth factor 21: a novel metabolic regulator with potential therapeutic properties in obesity/type 2 diabetes mellitus. Physiol Res 2009;58:1-7.

7 Kharitonenkov A, Shanafelt AB. Fibroblast growth factor-21 as a therapeutic agent for metabolic diseases. BioDrugs 2008;22:37-44.

8 Xu J, Lloyd DJ, Hale C, et al. Fibroblast growth factor 21 reverses hepatic steatosis, increases energy expenditure, and improves insulin sensitivity in diet-induced obese mice. Diabetes 2009:58:250-9.

9 Coskun T, Bina HA, Schneider MA, et al. Fibroblast growth factor 21 corrects obesity in mice. Endocrinology 2008;149:6018-27.

10 Uonaga T, Toyoda K, Okitsu T, et al. FGF-21 enhances islet engraftment in mouse syngeneic islet transplantation model. Islets 2010;2:247-51.

11 Kharitonenkov A, Wroblewski VJ, Koester A, et al. The metabolic state of diabetic monkeys is regulated by fibroblast growth factor-21. Endocrinology 2007;148:774-81.

12 Moher D, Liberati A, Tetzlaff J, et al. Preferred reporting items for systematic reviews and meta-analyses: the PRISMA statement. J Clin Epidemiol 2009;62:1006-12.

13 Robert K, Pagès C, Ledru A, et al. Regulation of extracellular signal-regulated kinase by homocysteine in hippocampus. Neuroscience 2005:133:925-35.

14 Wang P, Mao YM, Zhao CN, et al. Increased pulse wave velocity in systemic lupus erythematosus: a meta-analysis. Angiology 2018:69:228-35.

15 Higgins JP, Thompson SG. Quantifying heterogeneity in a meta-analysis. Stat Med 2002;21:1539-58.

16 Higgins JP, Thompson SG, Deeks JJ, et al. Measuring inconsistency in meta-analyses. BMJ 2003;327:557-60.

17 Egger M, Davey Smith G, Schneider M, et al. Bias in meta-analysis detected by a simple, graphical test. BMJ 1997;315:629-34.

18 Akour A, Kasabri V, Boulatova N, et al. Levels of metabolic markers in drug-naive prediabetic and type 2 diabetic patients. Acta Diabetol 2017;54:163-70.

19 Esteghamati A, Momeni A, Abdollahi A, et al. Serum fibroblast growth factor 21 concentrations in type 2 diabetic retinopathy patients. Ann Endocrinol 2016;77:586-92.

20 Shafaei A, Khoshnia M, Marjani A. Serum level of fibroblast growth factor 21 in type 2 diabetic patients with and without metabolic syndrome. Journal of Medical Sciences 2015;15:80-6

21 Lin Y, Xiao YC, Zhu H, et al. Serum fibroblast growth factor 21 levels are correlated with the severity of diabetic retinopathy. J Diabetes Res 2014;2014:1-6.

22 Xiao Y, Xu A, Law LS, et al. Distinct changes in serum fibroblast growth factor 21 levels in different subtypes of diabetes. J Clin Endocrinol Metab 2012;97:E54-E58.

23 Wang $B$, Yang $G$, Yang $M$, et al. Mitiglinide treatment may decreases plasma fibroblast growth factor-21 levels in individuals with new-onset T2DM. Cytokine 2012;57:300-3.

24 Jian W-X, Peng W-H, Jin J, et al. Association between serum fibroblast growth factor 21 and diabetic nephropathy. Metabolism 2012;61:853-9.

25 Cheng X, Zhu B, Jiang F, et al. Serum FGF-21 levels in type 2 diabetic patients. Endocr Res 2011;36:142-8.

26 Li K, Li L, Yang M, et al. Effects of rosiglitazone on fasting plasma fibroblast growth factor-21 levels in patients with type 2 diabetes mellitus. Eur J Endocrinol 2009; 161:391-5

27 Chen WW, Li L, Yang GY, et al. Circulating FGF-21 levels in normal subjects and in newly diagnose patients with type 2 diabetes mellitus. Exp Clin Endocrinol Diabetes 2008:116:65-8.

28 Li L, Yang G, Ning H, et al. Plasma FGF-21 levels in type 2 diabetic patients with ketosis. Diabetes Res Clin Pract 2008;82:209-13.

29 Palma S, Delgado-Rodriguez M. Assessment of publication bias in meta-analyses of cardiovascular diseases. J Epidemiol Community Health 2005;59:864-9.

30 Sánchez J, Palou A, Picó C. Response to carbohydrate and fat refeeding in the expression of genes involved in nutrient partitioning and metabolism: striking effects on fibroblast growth factor-21 induction. Endocrinology 2009;150:5341-50.

31 Kharitonenkov A, Shiyanova TL, Koester A, et al. FGF-21 as a novel metabolic regulator. J Clin Invest 2005:115:1627-35. 